\title{
Elliptic flow in heavy ion collisions near the balance energy
}

\author{
Yu-Ming Zheng ${ }^{\mathrm{a} *}$, C. M. Ko ${ }^{\mathrm{a}}$, Bao-An $\mathrm{Li}^{\mathrm{c}}$, and Bin Zhang ${ }^{\mathrm{a}}$ \\ ${ }^{a}$ Cyclotron Institute and Physics Department, Texas A\&M University \\ College Station, Texas 77843-3366, USA \\ ${ }^{\mathrm{c}}$ Department of Chemistry and Physics, Arkansas State University \\ P.O. Box 419, State University, AR 72467-0419, USA
}

\begin{abstract}
The proton elliptic flow in collisions of ${ }^{48} \mathrm{Ca}$ on ${ }^{48} \mathrm{Ca}$ at energies from 30 to $100 \mathrm{MeV} /$ nucleon is studied in an isospin-dependent transport model. With increasing incident energy, the elliptic flow shows a transition from positive to negative flow. Its magnitude depends on both the nuclear equation of state (EOS) and the nucleon-nucleon scattering cross section. Different elliptic flows are obtained for a stiff EOS with free nucleon-nucleon cross sections and a soft EOS with reduced nucleon-nucleon cross sections, although both lead to vanishing in-plane transverse flow at the same balance energy. The study of both in-plane and elliptic flows at intermediate energies thus provides a means to extract simultaneously the information on the nuclear equation of state and the nucleon-nucleon scattering cross section in medium.

PACS number(s): 25.70.-z, 25.75.Ld, 24.10.Lx
\end{abstract}

Heavy ion collisions provide the possibility to study the properties of nuclear matter in conditions vastly different from that in normal nuclei, such as high density and excitation as well as large difference in the proton and neutron numbers [1 5]. Such knowledge is not only of interest in itself but also useful in understanding astrophysical phenomena such as the properties of the core of compact stars, the evolution of the early universe, and the formation of elements in stellar nucleosynthesis. One observable that has been extensively used for extracting such information from heavy ion collisions is the collective flow of various particles [6 21] (for a recent review, see Refs. [22 25]). For example, the proton flow in heavy ion collisions at $200 \mathrm{MeV} /$ nucleon to $1 \mathrm{GeV} /$ nucleon has been found to be consistent with a soft nuclear equation of state [7,8]. From the kaon flow in heavy ion collisions at 1 to 2 $\mathrm{GeV} /$ nucleon, the existence of a weak repulsive kaon potential has been obtained [10]. In heavy ion collisions at energies higher than $2 \mathrm{GeV} /$ nucleon, recent studies of proton flow seem to indicate that there is a softening of nuclear equation of state as the nuclear density and excitation increase [18]. There are also suggestions that particle collective flows at

\footnotetext{
*Permanent address: China Institute of Atomic Energy, P. O. Box 275(18), Beijing 102413, China
} 
ultrarelativistic heavy ion collisions are sensitive to the initial parton dynamics [19] and subsequent phase transitions [17,20,21].

In general, collective flow in heavy ion collisions is affected by both the nuclear meanfield potential and nucleon-nucleon cross sections. In heavy ion collisions at intermediate energies of a few tens $\mathrm{MeV} /$ nucleon, the collision dynamics is dominated by the attractive nuclear mean-field potential as nucleon-nucleon scatterings are largely blocked due to the Pauli principle. As a result, the nucleon transverse flow in the reaction plane is negative, i.e., nucleons moving in the projectile direction are deflected to negative angles. With increasing incident energies, the repulsive nucleon-nucleon scattering becomes important and reduces the negative flow caused by the attractive nuclear mean-field potential. At certain incident energy, called the balance energy, in-plane transverse flow vanishes as a result of the cancellation between these two competing effects [26]. The disappearance of transverse collective flow has been experimentally observed in heavy ion collisions [27 29]. The measured balance energy depends strongly on the mass and isospin of colliding nuclei as well as on the impact parameter of collisions [22,23,27, 29]. Studies based on transport models have shown that the same balance energy can be obtained with different nuclear equations of state and inmedium nucleon-nucleon scattering cross sections [30,31]. To extract their information from measured balance energies thus requires the measurement of other observables. One of the present authors [32] has recently shown that different EOS and cross sections which give the same balance energy show different differential transverse flows, i.e., their transverse flows have different dependence on the total transverse momentum. In the present paper, we shall study instead the proton elliptic flow, which measures the anisotropy in their transverse momentum distribution. In particular, using the isospin-dependent Boltzmann-UehlingUhlenbeck (IBUU) model [31], we shall consider collisions of ${ }^{48} \mathrm{Ca}+{ }^{48} \mathrm{Ca}$ at energies from 30 to $100 \mathrm{MeV} /$ nucleon. As shown below, different EOS and cross sections that give the same balance energy lead to significantly different elliptic flows.

Taking the beam direction along the $z$-axis and the reaction plane on the $x-z$ plane, the elliptic flow is then determined from the average difference between the square of the $x$ and $y$ components of particle transverse momentum, i.e.,

$$
v_{2}=\left\langle\frac{p_{x}^{2}-p_{y}^{2}}{p_{x}^{2}+p_{y}^{2}}\right\rangle .
$$

It corresponds to the second Fourier coefficient in the transverse momentum distribution 24,33 and describes the eccentricity of an ellipse-like distribution, i.e., $v_{2}>0$ indicates in-plane enhancement, $v_{2}<0$ characterizes the squeeze-out perpendicular to the reaction plane, and $v_{2}=0$ shows an isotropic distribution in the transverse plane.

The IBUU transport used in the present study treats explicitly protons and neutrons. It also includes an asymmetry term in the nuclear mean-field potential and different scattering cross sections for protons and neutrons. The nuclear mean-field potential is parameterized as

$$
\begin{gathered}
U\left(\rho, \tau_{z}\right)=U_{0}(\rho)+U_{\text {asy }}\left(\rho, \tau_{z}\right), \\
U_{0}(\rho)=a\left(\frac{\rho}{\rho_{0}}\right)+b\left(\frac{\rho}{\rho_{0}}\right)^{\sigma},
\end{gathered}
$$




$$
U_{\text {asy }}\left(\rho, \tau_{z}\right)=C \frac{\rho_{p}-\rho_{n}}{\rho_{0}} \tau_{z}
$$

In the above, $\rho_{0}$ is the normal nuclear matter density; $\rho, \rho_{n}$ and $\rho_{p}$ are the nucleon, neutron, and proton densities, respectively; and $\tau_{z}$ equals 1 for proton and -1 for neutron. For the strength of the asymmetry potential, we take $C=32 \mathrm{MeV}$. Two different EOS are used in our studies: a stiff EOS with compressibility of $380 \mathrm{MeV}(a=-124 \mathrm{MeV}, b=70.5$ $\mathrm{MeV}, \sigma=2)$ and a soft one with compressibility of $200 \mathrm{MeV}(a=-356 \mathrm{MeV}, b=303$ $\mathrm{MeV}, \sigma=7 / 6)$. We also include the Coulomb potential for protons. For nucleon-nucleon scatterings, both elastic and inelastic channels are included by using the experimentally measured cross sections with explicit isospin dependence. Details of the IBUU model can be found in Refs. [31].

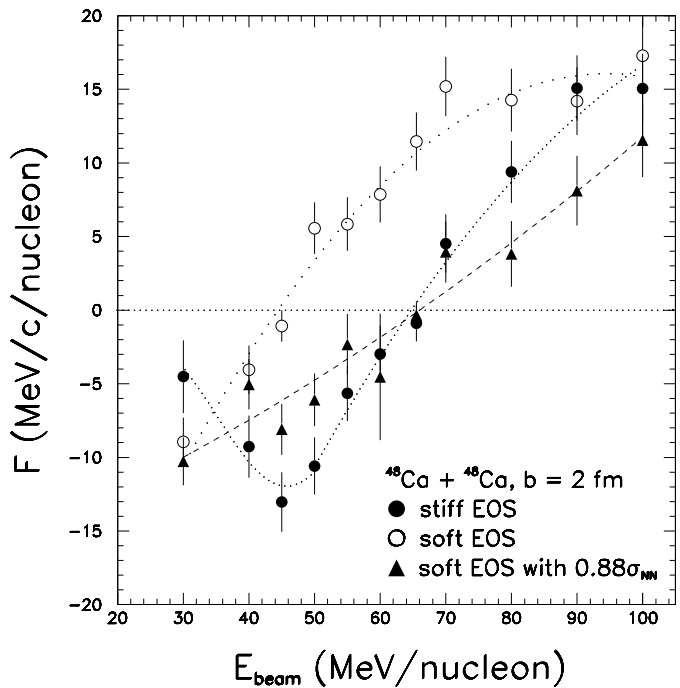

FIG. 1. The excitation function of the proton flow parameter in ${ }^{48} \mathrm{Ca}+{ }^{48} \mathrm{Ca}$ collisions at an impact parameter of $2 \mathrm{fm}$. Open and solid circles correspond to soft and stiff EOS, respectively. Solid triangles are from the soft EOS and a reduced nucleon-nucleon cross section $0.88 \sigma_{N N}$.

We first study the flow parameter at midrapidity, which is defined by

$$
F=\left.\frac{d<p_{x}>}{d y}\right|_{y=0}
$$

In Fig. 1, we show the incident energy dependence of the proton flow parameter in ${ }^{48} \mathrm{Ca}$ $+{ }^{48} \mathrm{Ca}$ reactions at an impact parameter of $2 \mathrm{fm}$. Open circles are obtained from the IBUU model using the soft EOS. In this case, the proton in-plane transverse flow below about $45 \mathrm{MeV} /$ nucleon is negative as a result of the dominant effect of attractive nuclear mean-field potential. Above this incident energy, nucleon-nucleon scatterings become more important, and their repulsive effects lead to a positive flow parameter. For the stiff EOS, shown by solid circles, the flow parameter is generally reduced because of a less attractive mean-field potential than that for the soft EOS. The exception to this general behavior 
occurs, however, at very low incident energies below about $40 \mathrm{MeV} /$ nucleon, where the flow parameter increases instead with decreasing incident energy. This is due to the fact that scattering effects at low energies are not strong enough to reverse the effect due to the attractive mean-field potential. We have also shown in Fig. 11 by solid triangles the flow parameter obtained for the soft EOS but with the nucleon-nucleon scattering cross section reduced by $12 \%$. Compared with the case of the soft EOS and free nucleon-nucleon cross section $\sigma_{N N}$, the flow parameter is reduced as expected. We note that the same balance energy, about $65.5 \mathrm{MeV} /$ nucleon, is obtained for both the stiff EOS with $\sigma_{N N}$ and the soft EOS with $0.88 \sigma_{N N}$.

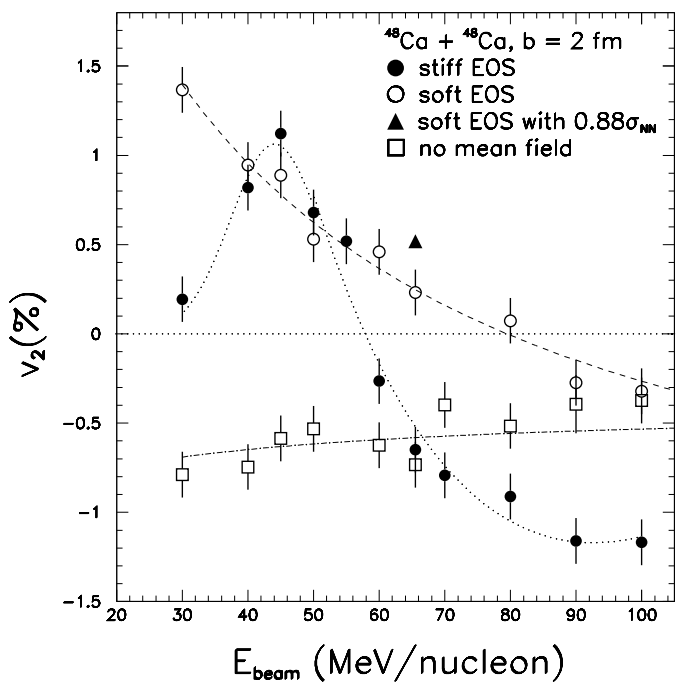

FIG. 2. The excitation function of the proton elliptic flow in ${ }^{48} \mathrm{Ca}+{ }^{48} \mathrm{Ca}$ collisions at an impact parameter of $2 \mathrm{fm}$.

The excitation function of the proton elliptic flow for the same reaction is shown in Fig. 2. For both the soft EOS (open circles) and the stiff EOS (solid circles) the proton elliptic flow changes from positive flow at low energies to negative flow at high energies, i.e., a transition from the dominance of in-plane transverse flow to that of out-of-plane squeeze out as the beam energy increases. However, the energy at which this transition occurs differs for the two EOS; it is smaller for the stiff EOS than for the soft EOS. To understand this difference, we also show in Fig. 2 the proton elliptic flow in the absence of mean-field potential (open squares), which is negative at all energies, i.e., out-of-plane squeeze out dominates over inplane transverse flow. Since the soft EOS gives a larger in-plane transverse flow than that due to the stiff EOS, it leads to a higher energy at which the elliptic flow changes sign. The abnormal behavior at energies below $45 \mathrm{MeV} /$ nucleon, where the elliptic flow for the stiff EOS decreases with decreasing energy, reflects its behavior in the flow parameter as shown in Fig. 11.

Also shown in Fig. 2 is the elliptic flow for the soft EOS with $0.88 \sigma_{N N}$ (solid triangle), which gives the same flow parameter as the stiff EOS with $\sigma_{N N}$. As seen, the two give very different elliptic flow; it is negative for the stiff EOS with $\sigma_{N N}$ but is positive for the soft 
EOS with $0.88 \sigma_{N N}$.

In summary, the IBUU model has been used to study the proton elliptic flow in collisions of ${ }^{48} \mathrm{Ca}+{ }^{48} \mathrm{Ca}$ at an impact parameter of $2 \mathrm{fm}$ for beam energies from 30 to 100 $\mathrm{MeV} /$ nucleon. We find that it shows a transition from positive to negative flow as the incident energy increases. A strong dependence on both the nuclear EOS and the nucleonnucleon cross section is seen in proton elliptic flow. Although both the stiff EOS with $\sigma_{N N}$ and the soft EOS with $0.88 \sigma_{N N}$ have the same balance energy, they are found to give very different elliptic flows. The study of both in-plane and elliptic flows at intermediate energies thus allows one to extract simultaneously the information on the nuclear equation of state and the nucleon-nucleon scattering cross section in medium.

We thank Joe Natowitz for a critical reading of the manuscript. This work was supported in part by the National Science Foundation Grant PHY-9870038, the Department of Energy grant DE-FG03-93ER40773, the Welch Foundation Grant A-1358, and the Texas Advanced Research Program FY97-010366-068. 


\section{REFERENCES}

[1] H. Stöcker and W. Greiner, Phys. Rep. 137, 277 (1986).

[2] W. Cassing, V. Metag, U. Mosel, and K. Niita, Phys. Rep. 188, 363 (1990).

[3] J. Harris and B. Müller, Ann. Rev. Nucl. Part. Sci. 46, 71 (1996).

[4] C. M. Ko and G. Q. Li, J. Phys. G 22, 1673 (1996); C. M. Ko, V. Koch, and G. Q. Li, Ann. Rev. Nucl. Part. Sci. 47, 509 (1997).

[5] B. A. Li, C. M. Ko and W. Bauer, Int. J. Mod. Phys., E 7, 147 (1998).

[6] P. Danielewicz and G. Odyniec, Phys. Lett. B 157, 146 (1985).

[7] Q. B. Pan and P. Danielewicz, Phys. Rev. Lett. 70, 2062 (1993).

[8] J. Zhang, S. Das Gupta and C. Gale, Phys. Rev. C 50, 1617 (1994).

[9] B. A. Li, Phys. Lett. B 300, 14 (1993); Nucl. Phys. A 570, 797 (1994).

[10] G. Q. Li, C. M. Ko, and B. A. Li, Phys. Rev. Lett. 74, 235 (1995); G. Q. Li and C. M. Ko, Nucl. Phys. A 594, 460 (1995); Phys. Rev. C 54, 1897, R2159 (1996).

[11] Z. S. Wang et al., Nucl. Phys. A645, 177 (1999).

[12] G. Q. Li and G. E. Brown, Nucl. Phys. A 636, 487 (1998).

[13] B. A. Li, C. M. Ko, and G. Q. Li, Phys. Rev. C 54, 844 (1996); B. A. Li and C. M. Ko, Phys. Rev. C 53, R22 (1996); C 54, 3283 (1996); C 58, R1382 (1998).

[14] B. A. Li, B. Zhang, A. T. Sustich, and C. M. Ko, Phys. Rev. C, in press, nuclth/9906049; B. A. Li, C. M. Ko, A. T. Sustich, and B. Zhang, ibid., in press, nuctth/9904013.

[15] C. M. Hung and E. V. Shuryak, Phys. Rev. Lett. 75, 4003 (1995).

[16] D. H. Rischke and M. Gyulassy, Nucl. Phys. A 597, 701 (1996).

[17] H. Sorge, Phys. Rev. Lett. 78, 2309 (1997); ibid. 82, 2048 (1999).

[18] P. Danielewicz et al., Phys. Rev. Lett. 81, 2438 (1998).

[19] B. Zhang, M. Gyulassy, and C. M. Ko, Phys. Lett. B, in press; nucl-th/9902010.

[20] H. Heiselberg and A. Levy, Phys. Rev. C 59, 2716 (1999).

[21] D. Teney and E. V. Shuryak, nucl-th/9904006.

[22] G. D. Westfall and J. Péter, Ann. Rev. Nucl. Part. Sci., in press.

[23] T. C. Awes, Nucl. Phys. A 630, 499c (1998).

[24] J.-Y. Ollitrault, Nucl. Phys. A 638, 195c (1998).

[25] W. Reisdorf, Nucl. Phys. A 630 (1998) 15c; W. Reisdorf and H. G. Ritter, Ann. Rev. Nucl. Part. Sci. 47, 663 (1997).

[26] S. Das Gupta and G. D. Westfall, Phys. Today, 46, 34 (1993).

[27] J. P. Sullivan et al., Phys. Lett. B 249, 8 (1990).

[28] G. D. Westfall et al., Phys. Rev. Lett. 71, 1986 (1993); M. J. Huang, et al., ibid. 77, 3739 (1996); Nucl. Phys. A 630, 27c (1998).

[29] J. Péter et al., Nucl. Phys. A 545, 173c (1992); Z. Y. He et al., ibid. 598, 248 (1996); A. Buta et al., ibid. 584, 397 (1995); J. C. Angélique et al., ibid. 614, 261 (1997).

[30] D. Klakow et al., Phys. Rev. C 48, 1982 (1993).

[31] B. A. Li, Z. Z. Ren, C. M. Ko, and S. J. Yennello, Phys. Rev. Lett. 76, 4492 (1996); B. A. Li, C. M. Ko and Z. Z. Ren, Phys. Rev. Lett. 78 , 1644 (1997).

[32] B. A. Li and A. T. Sustich, Phys. Rev. Lett. 82, 5004 (1999).

[33] S. A. Voloshin, Phys. Rev. C 55, R1630 (1997); A. M. Poskanzer and S. A. Voloshin, ibid C 58, 1671 (1998). 